\title{
Effects of Vitamin B12 on Performance and Circadian Rhythm in Normal Subjects
}

\author{
Geert Mayer, M.D., Margarete Kröger, M.D., and Karlheinz Meier-Ewert, M.D.
}

This preliminary study investigates effects of methyl-and cyanocobalamin on circadian rhythms, well-being, alertness, and concentration in healthy subjects. Six women (mean age 35 years) and 14 men (mean age 37 years) were randomly assigned to treatment for 14 days with $3 \mathrm{mg}$ cyano-(CB12) or methylcobalamin (MB12) after 9 days of pre-treatment observation. Levels in the CB12 group increased rapidly in the first, then slowly in the second treatment week, whereas increase in the MB12 group was linear. Urinary aMT6s excretion was reduced by both forms of vitamin B12 over 24 hours with a significant decrease between 0700-1100 hours, whereas urinary excretion of potassium was significantly increased between 0700-1100 hours. Activity from 2300-0700 hours increased significantly under both forms of vitamin B12. Sleep time was significantly reduced under MB12 intake. In this group the change in the visual analogue scales items "sleep quality," "concentration," and "feeling refreshed" between pretreatment and the first week of treatment showed significant correlations with vitamin B12 plasma levels. Cortisol excretion and temperature were not affected by either medication. We conclude that vitamin $B 12$ exerts a direct influence on melatonin. Only MB12 has a positive psychotropic alerting effect with a distribution of the sleepwake cycle toward sleep reduction. (C) 1996 American College of Neuropsychopharmacology [Neuropsychopharmacology 15:456-464, 1996]
KEY WORDS: Methylcobalamin; Cyanocobalamin; Urinary melatonin and potassium excretion; Visual analogue scales; Vigilance; Sleep quality; Circadian rhythm

In recent years, numerous authors have reported the therapeutic benefits of vitamin B12 in treating sleepwake disorders (Kamgar-Parsi et al. 1983; Okawa et al. 1990; Ohta et al. 1991; Takahashi 1992; Yamazaki et al. 1991; Maeda et al. 1992). Most of these authors used the activated form of vitamin B12, methylcobalamin (Esai Company), which is not available in Europe. In animal experiments, it shows a better transport to organelles within nerve cells than cyanocobalamin. Preliminary re-

From the Sleep Disorder Unit, Hephata Klinik, SchwatmstadtTreysa, Germany.

Address correspondence to: Dr. Geert Mayer, Sleep Disorder Unit, Hephata Klinik, Schimmelpfengstrasse, 34613 SchwalmstadtTreysa, Germany.

Received September 19, 1995; revised January 19, 1996; accepted January 26, 1996. sults of a Japanese Multicenter study (Takahashi 1992) show that two-thirds of 75 patients with sleep-wake disorders benefit from methylcobalamin medication at doses ranging from 1.5 to $3.0 \mathrm{mg}$.

Vitamin B12 seems to exert influence on biological rhythms by shortening the length of sleep-wake rhythm, improving the entrainment of the endogenous sleepwake cycle to the environmental 24-hour rhythm (Okawa et al. 1990), and by affecting the circadian component of sleep propensity (Ohta et al. 1991). In addition vitamin B12 has been reported to increase light sensitivity by affecting melatonin secretion (Yamazaki et al. 1991; Honma et al. 1992).

In a study using healthy subjects, Honma et al. (1992) found a significant phase advance of the melatonin rhythm plus a decreased mean 24-hour plasma melatonin level comparing vitamin B12 application with placebo condition. Yamazaki et al. (1991) found no phase change of circadian rhythms in healthy subjects. They reported improvements in vigilance and concentration 
and, in some cases, mood. Using a constant routine procedure with five healthy male subjects, Uchiyama et al. (1995) found that vitamin B12 improved alertness.

This study was carried out in order to determine whether to use the cyanocobalamin or methylcobalamin form of vitamin B12 in further planned experiments of circadian rhythm disorders. We used a between-subjects design with random assignment of healthy subjects to one of the two treatment conditions and tested effects on circadian rhythms, well-being, concentration, and alertness. Due to the preliminary character of the study, we decided against the inclusion of a control group.

\section{METHODS}

\section{Subjects}

Six healthy women (mean age 35.17 years) and 14 men (mean age 37.2 years) of our clinic staff participated in the study after giving their informed consent. Exclusion criteria included disorders of excessive daytime sleepiness, chronic intake of any kind of medication, fasting, infectious diseases, diarrhea, chronic consumptive diseases, vegetarian diet, pregnancy and breastfeeding, disorders of gastrointestinal resorption (including Billroth), oral or intravenous administration of vitamin B12 within the last 3 months and shift work beyond 2100 hours. Subjects with actigraphic variations of the rest activity cycle of more than 1 hour daily (except for weekends) were excluded.

Subjects were randomly assigned to either the cyanocobalamin (CB12) or methylcobalamin (MB12) group in a single-blind fashion. There was no placebo control group. Mean age was $36.6 \pm 5.2$ years in the CB12 group and $36.2 \pm 5.2$ years in the MB12 group. One male participant in the MB12 group was excluded for noncompliance. Participants were asked to avoid excessive physical activity (Monteleone et al. 1990), excessive consumption of alcohol and food, and to keep regular sleep-wake habits (as determined by a diary kept the week before the study). They were allowed to sleep 2 hours longer on weekends. Four subjects (two women in the CB12, one woman and one man in the MB12 group) were on shift duty. They kept the same shift one week before the beginning and throughout the study (daytime work from 0600-1400 hours and 1300-2100 hours).

\section{Study Design}

Study length was 23 days -9 days of pretreatment and 14 days of intake of vitamin B12 3mg at 0700 hours (Table 1). The study was conducted in December and January with darkness prevailing from 1703-0826 hours (range 1616-1713, 0804-0826 hours with two lux at dusk and dawn) in the geographic area. On weekdays, participants were mainly exposed to artificial light inside and outside the hospital (inside hospital range 0500-0575 lux).

\section{Methods}

Cole et al. (1992) and Sadeh et al. (1989) used actigraphy to distinguish sleep and wake. With their actigraphs and algorhythms they found an accuracy rate of $86.2 \%$ to $87.9 \%$ in normal subjects. We used actigraphs by Firma ZAK Simbach, Germany. The one axis piezo acceleration recorders have a zero-crossing, nonevent mode, counting 0 to 240 impulses every 2 to 3 minutes at a minimal sensitivity of $0.1 \mathrm{~g}$ and were worn on the wrist of the nondominant hand. Well-being was rated by visual analogue scales (VAS) 0 to $100 \mathrm{~mm}$ filled out twice daily. The morning VAS covered patients' feeling fresh-tired, having had a good night-bad night, their feeling excited-calm plus their estimate of night sleep duration. The evening VAS assessed their feeling freshtired and unconcentrated-concentrated.

On days 1 and 2, 8 and 9, 15 and 16, 22 and 23, we measured rectal temperature (Wever 1979; Czeisler et al. 1986) on a 2- or 3-minute basis (Firma ZAK), the plasma levels of vitamin B12 (drawn at 0800 hours) and

Table 1. Study Design

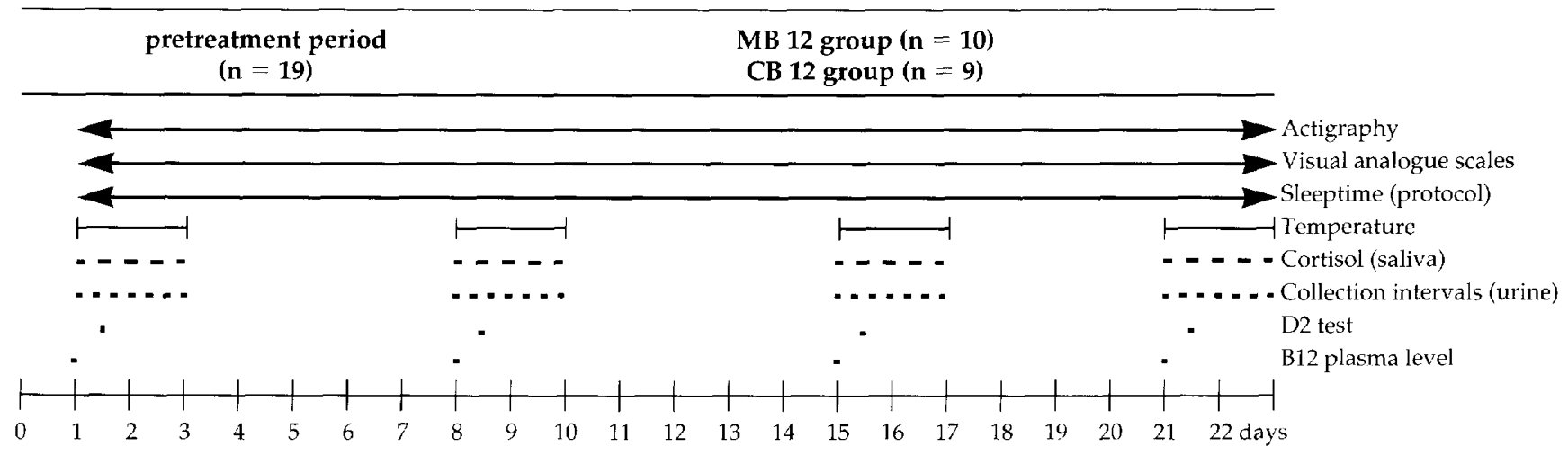


cortisol (RIA Firma Hermann Behrmann, Bad Nauheim, Germany) via salivettes at 0700 hours and 1800 hours (Al-Ansari et al. 1982; Laudat et al. 1988). Urine was collected from 0700-1100, 1100-1500, 1500-1900, 1900-2300 and 2300-0700 hours for evaluation of 6-sulphatoxymelatonin $=$ aMT6 (Aldhous and Arendt 1988) and potassium and sodium as other markers of the endogenous circadian oscillator. To determine concentration, two consecutive $\mathrm{d} 2$ tests (measuring concentration and speed by visually identifying given items and erasing them) were performed collectively at 1200 hours. The score comprised total number of reactions $(600) \mathrm{mi}-$ nus wrong reactions.

Sleep was evaluated according to a software supplied by the manufacturer (ZAK) of the actigraph using a three-threshold paradigm (Pollmächer and Schulz 1987). Threshold for sleep was set at 6 counts/minute, 14 counts/minute for awakening and 20 counts/minute for sleep disruption for at least 2 minutes. Given the instructions concerning food and beverage consumption as well as excessive activity, we felt that four repeated measurements for all parameters over 48 hours were tolerable for the subjects and would suffice to indicate changes in the circadian parameters despite masking effects, i.e., the product of changes in the activity of the subject that is superimposed on an endogenous circadian rhythm.

\section{Statistical Methods}

The SPSS program package was used to calculate the following statistics: two-tailed $t$-test, analysis of variance and covariance for repeated measurements, and Spearman's correlation ranks. For all tests, the means of days $1,2,8$, and 9 were determined as pretreatment. $T$-tests, performed for independent groups, were automatically tested for inhomogeneity of variance. In this case $t$-test values and the respective probabilities for "separate variance estimate" are referred to in the results. In case of homogeneous variance, the values are listed for "pooled variance estimate." The F-values for testing of inhomogeneity of variance are not listed explicitly.

Routine testing of repeated measurements of analy-

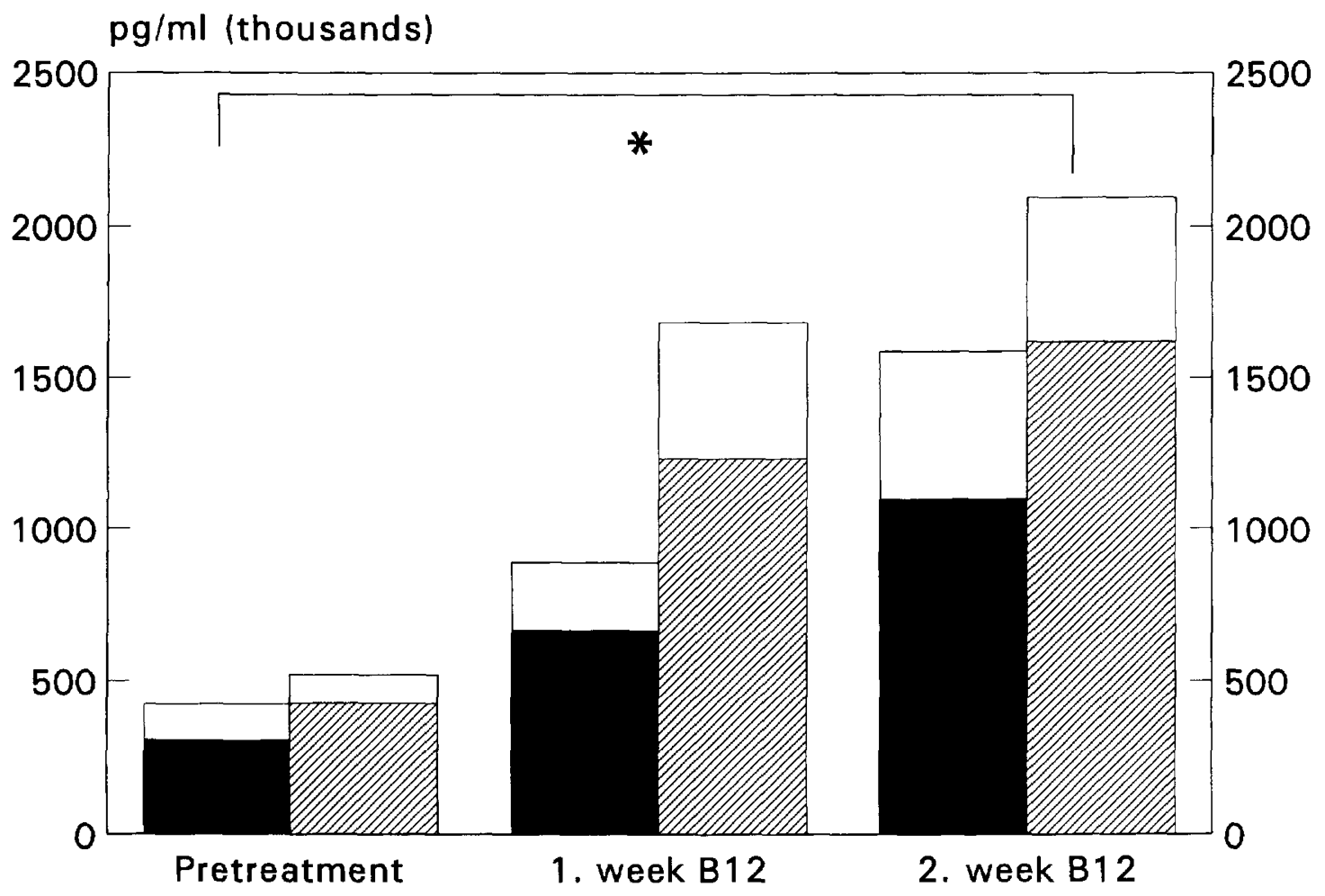

$$
\begin{aligned}
& \mathbf{M}=\mathbf{M B} 12 \\
& \mathbf{( n = 8 )}(\mathbf{n}=\mathbf{9})
\end{aligned}
$$

Figure 1. Mean vitamin B12 plasma levels before and during vitamin B12 treatment. Black bars represent mean vitamin B12 plasma levels of the MB12 group, hatched bars those of the CB12 group, white bars represent standard error during pretreatment (days 1,2,8,9) and during first and second week of vitamin B12 treatment. Anova revealed a significant time effect $(F=$ $41,38, p=0,000)$ and a significant group effect $(F=7,48, p=0,016)$ on the difference as indicated by asterisk. 
sis of variance was performed for inhomogeneity of variance (Bartlett-Box-Test). Our data never contained inhomogeneous variances. For independent variables analysis of variance for comparison of groups of different sizes automatically interpolated to a common group size. For dependent variables, cell size is automatically identical.

Temperature acrophase, mesor, and nadir were calculated by fitting a curve to the plotted raw data, using the locally weighted least squared error method, which weights the data to find the best smooth curve through the plot. Statistically smoothing factor was determined to be $5 \%$ for a 48 -hour record (program according to manual of Kaleida Graph I, version 2.0.2 by Abelbeck software).

\section{Pre-treatment Differences in Laboratory Parameters}

During pretreatment, the CB12 group $(n=10)$ differed from the MB12 group $(n=9)$ by a higher mean of vita$\mathrm{min}$ B12 levels $(437 \pm 14.9$ vs $311.2 \pm 18.6 \mathrm{pg} / \mathrm{ml}, p<$ $.0001)$, a longer mean total sleep time $(438.3 \pm 9.4$ vs
$392.1 \pm 8.9 \mathrm{~min}, p<.0001)$ and an earlier average midsleeptime (3:35 vs 3:12 hours, $p=\mathrm{NS}$ ).

The last two points suggest that the increased frequency of morning types in the CB12 group and of evening types (Horne et al. 1980) in the MB12 group was accidental. No differences were found in intraindividual temperature amplitude and acrophase and intraindividual sleep-wake habits except for weekends, which generally showed the expected delay of going to bed and getting up by almost 2 hours. Subjects on shift work did not get up or go to bed earlier or later compared with their previous shifts.

\section{RESULTS}

\section{Effects Common to Cyano- and Methylcobalamin}

Under treatment, mean vitamin B12 plasma levels increased significantly compared with the pretreatment period levels $(p<.001)$ (see Figure 1 ).

Urinary aMT6s excretion was measured at five collection intervals. Both forms of vitamin B12 triggered a

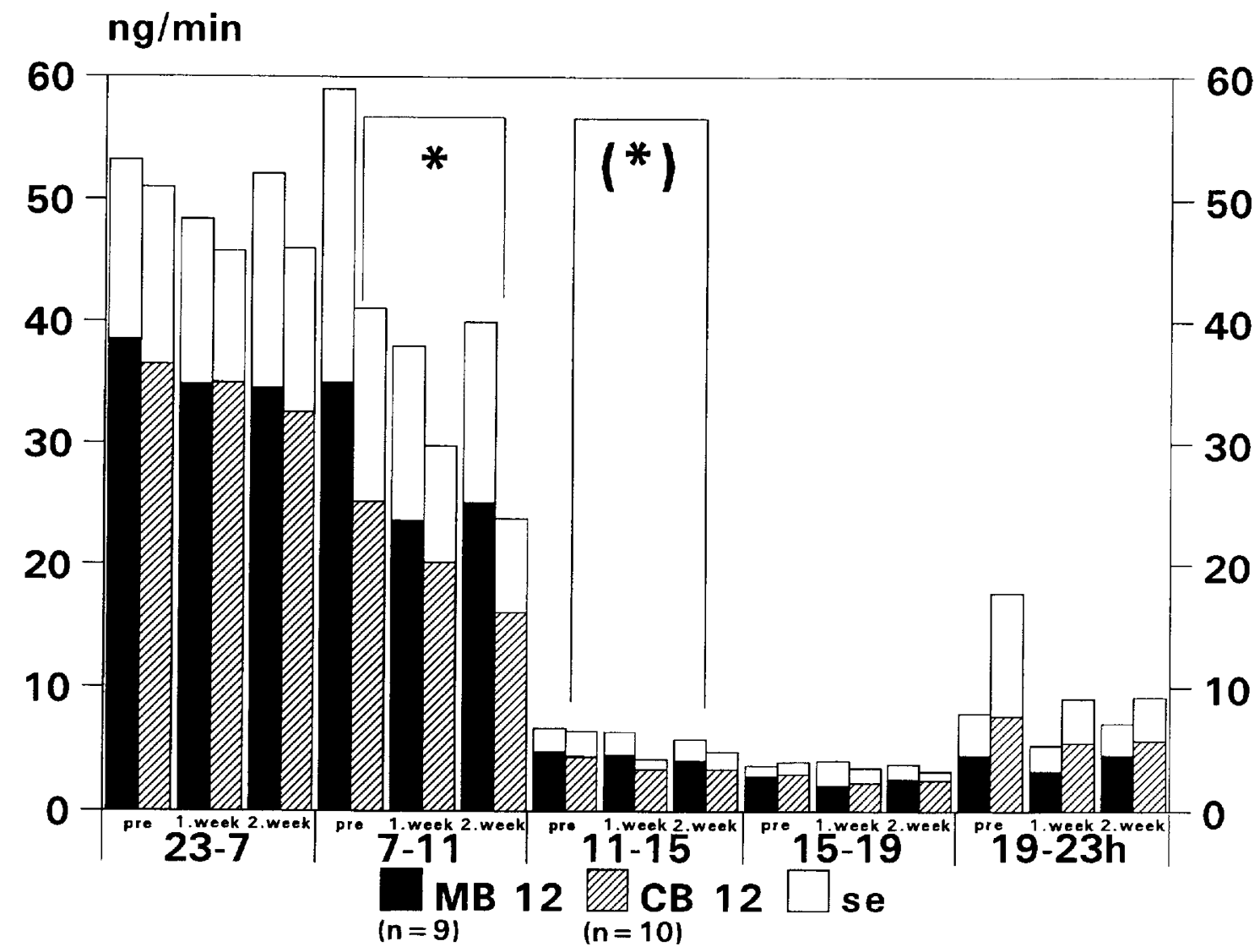

Figure 2. Mean urinary aMT6s excretion during 24 hours before and after vitamin B12 treatment. Black bars represent mean urinary aMT6s excretion at five consecutive intervals of the MB12 group, hatched bars those of the CB12 group, white bars represent standard error during pretreatment (pre = days 1,2,8,9) and during first and second week of vitamin B12 treatment. Anova revealed a significant time effect for MB12 and CB12 on the difference at the time interval 7-11 hours $(F=5,84, p=$ $0,007)$ and an almost significant time effect at the time interval $11-15$ hours $(F=2,73, p=0,08)$ as indicated by asterisks. 
significant decrease in the interval between 0700-1100 hours $(p<.007)$ (Figure 2) and a trend toward a significant decrease in the interval from 1100-1500 hours compared with pretreatment levels. For the majority of subjects, urinary aMT6s excretion peaked during the night, whereas only a few showed peak excretion between $0700-1100$ hours. No significant changes were recorded for the remaining collection periods.

Mean urinary potassium excretion increased nonsignificantly for all subjects between 0700-1100 hours during the second treatment week (Figure 3).

In the interval from 2300-0700 hours, mean actimeter activity increased significantly $(p<.039)$ (Figure 4$)$ between pre-treatment and the second treatment week.

Despite change of sleep duration, the mid-sleep time stayed stable in the MB12 (from 3:35 to 3:32 hours) and CB12 (from 3:12 to 3:16 hours) group.

In the $\mathrm{d} 2$ test, subjects in both groups had a signifi- cant increase $(p<.001)$ of the mean total score at each measurement. ANOVA revealed a significant increase of mean total score from the first to the second consecutive test $(p<.001)$. There was no difference between the groups.

\section{Differences between Cyano- and Methylcobalamin}

In the patients treated with cyanocobalamin, the increase of mean plasma vitamin B12 levels under treatment was (group by time $p<.044$ ) greater than in the MB12 group (Figure 1); mean urinary excretion of potassium increased in weeks 1 and 2 (Figure 3).

In the patients treated with methylcobalamin, sleep assessed by protocol $(p<.000)$ and by actimetry $(p<$ .036) was significantly shorter comparing pretreatment $(387.7 \pm 36$ vs $400.8 \pm 37.6$ minutes) with second treatment week ( $345.0 \pm 41.9$ vs $417 \pm 49.5$ minutes $)$, and

\section{$\mathrm{mol} / \mathrm{min}$}

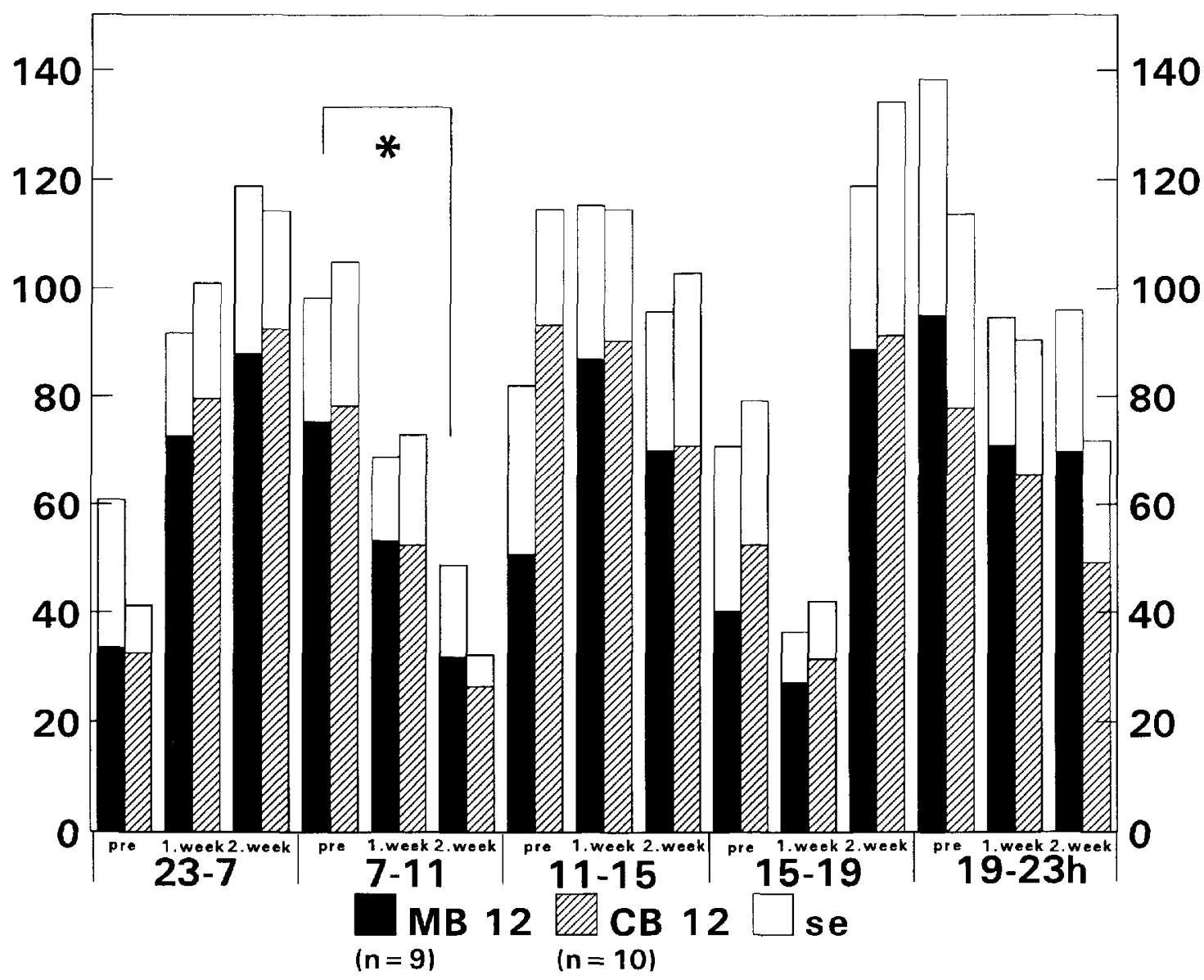

Figure 3. Mean urinary potassium excretion during 24 hours before and after vitamin B12 treatment. Black bars represent mean urinary potassium excretion at five consecutive intervals of the MB12 group, hatched bars those of the CB12 group, white bars represent standard error during pretreatment (pre = days $1,2,8,9$ ) and first and second week of vitamin B12 treatment. Anova revealed a significant group by time effect for MB12 and CB12 on the difference at the time interval 7-11 hours $(F=3,43, p=0,044)$ as indicated by asterisk. 


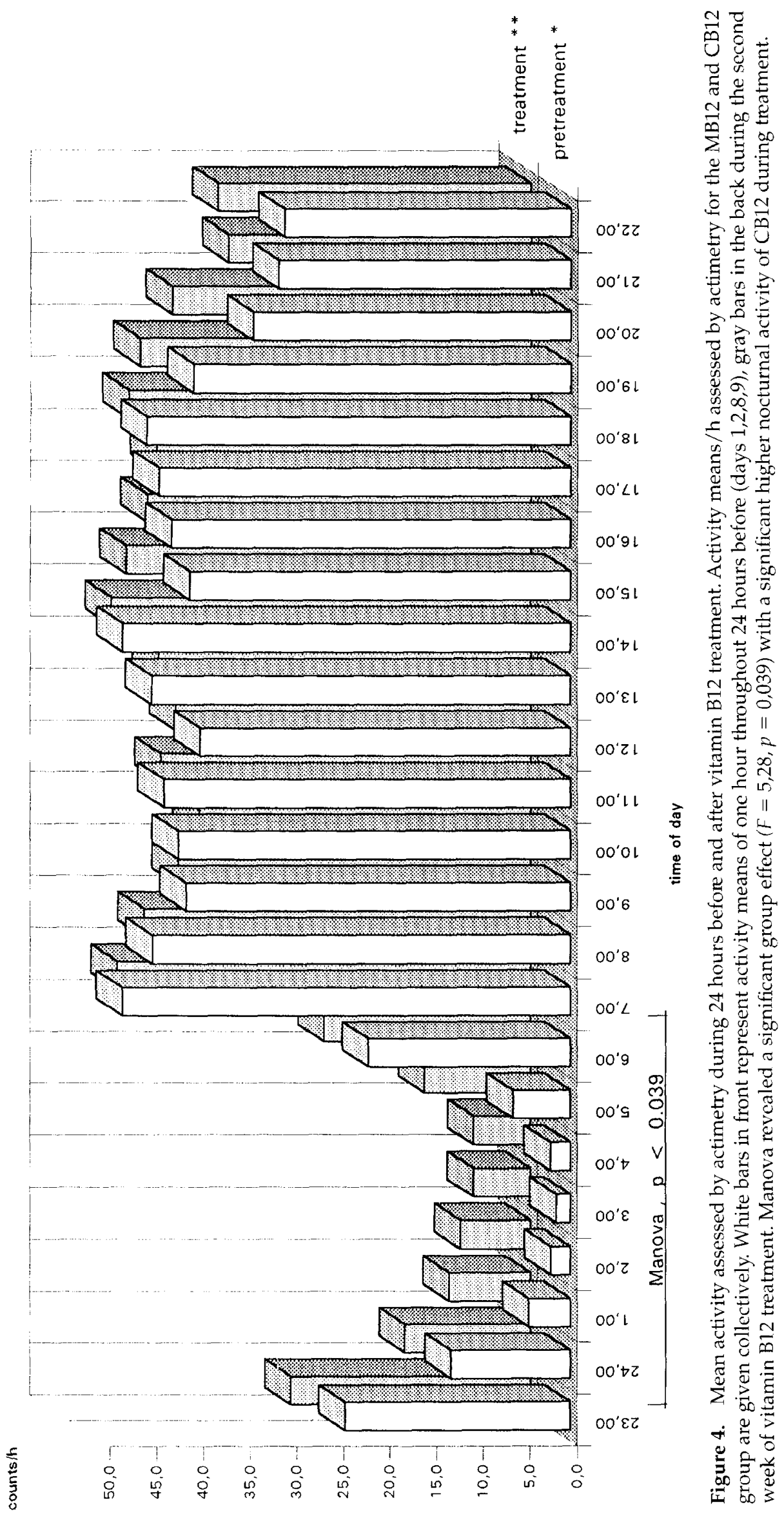


urinary potassium excretion decreased in the first treatment week.

\section{Alerting Properties of Methylcobalamin}

Table 2 gives correlations between vitamin B12 plasma levels and changes in the following VAS items:

- Methylcobalamin plasma levels correlated with change of the items "bad-good night" towards "good night sleep," "unconcentrated-concentrated" toward "concentration," "fresh-tired in the evening" "freshness" comparing pre-treatment with the first week of treatment.

- Cyanocobalamin plasma levels correlated with an increased rating of excitedness on the "excitedcalm" item of the VAS from the first to the second week of treatment.

Neither cyano- nor methylcobalamin caused any change in mean nocturnal urinary aMT6s excretion, mean urinary sodium excretion, amplitude of mean body core temperature, phase setting of the temperature minimum, morning and evening cortisol concentrations in saliva, mean diurnal activity as measured by actimetry, mean sleep length (actigraphy and protocol data).

Analysis of covariance showed that temperature changes are influenced by melatonin $(p<.001)$.

\section{DISCUSSION}

In this preliminary study, we investigated the difference between the effects of methyl- and cyanocobalamin on circadian rhythms, well-being, alertness, and concentration. The study design took into account the difficulties resulting from lack of a control group and masking effects, because we intended the study to be naturalistic and use its outcome for a further study to be performed under conditions of temporal isolation.

Urinary aMT6s and urinary potassium excretion, both relatively stable indicators of the circadian oscillator, were influenced by vitamin B12 treatment. The amplitude of mean urinary aMT6s was lowered (except for
1500-1900 hours), whereas the amplitude of mean urinary potassium was increased. These findings correspond with those of Okawa et al. (1990) and Honma et al. (1992). Significant changes for both urinary aMT6s and potassium in the morning may imply an increased sensitivity to morning light (even if artificial and low), faster decline of nocturnal urinary aMT6s, and increase of nocturnal urinary potassium excretion as a direct effect of vitamin B12. Because our nocturnal urinary collection intervals were too long, we were unable to find any change in urinary melatonin or potassium acrophase. The shortening of sleep time that we observed under MB12 treatment may support the hypotheses that vitamin B12 leads to a faster melatonin decline.

The differences in urinary aMT6s and potassium excretion between the two forms of vitamin B12 may depend on vitamin B12 plasma levels, which are close to saturation for the CB12 group and at a medium level for the MB12 group. If the effects are indeed vitamin B12 plasma level dependent, we would expect more pronounced effects at higher MB12 levels. Differences in urinary potassium need to be interpreted with caution, because potassium excretion is depending on nutrition, which we did not control. Souêtre et al. (1989), Waldhauser et al. (1990), and Dahlitz et al. (1991) found a high correlation between melatonin levels and sleepiness. We therefore expected that the suppression of daytime melatonin under vitamin B12 treatment would result in high levels of alertness. The vitamin B12-induced changes in visual analogue scales provide clear evidence of its alerting effects and correspond to the findings of Uchiyama et al. (1995). The findings of significantly improved night sleep and increased freshness and concentration throughout the day in the MB12 group correspond well with some of the objective measures, including a reduction of urinary aMT6 in the morning and shortened nocturnal sleep. These findings do not seem to be influenced by increased nocturnal motor activity. The correlations show a clear dose dependency of vitamin B12's effects on well-being. For the CB12 group, dose-dependent activation can only be found in the significant change of the item "excitedness" between the first to the second treatment weeks.

Table 2. Correlation Between Vitamin B12 Levels and Items of Visual Analogue Scales (VAS) Before and During Treatment

\begin{tabular}{llcccc}
\hline & & $\begin{array}{c}\text { Change of VAS between Pretreatment and } \\
\text { First Week of Treatment }\end{array}$ & $\begin{array}{c}\text { Change of VAS between First and Second } \\
\text { Week of Treatment }\end{array}$ \\
\hline \multirow{2}{*}{ morning } & & CB 12 group $n=10$ & MB 12 group $n=9$ & CB 12 group $n=10$ & MB 12 group $n=9$ \\
VAS r & VAS 1 fresh-tired & & & 0.58 & -0.60 \\
& VAS 2 bad-good night & -0.03 & $0.68^{*}$ & 0.02 & -0.13 \\
evening & VAS 3 excited-calm & -0.04 & 0.09 & $-0.70^{*}$ & 0.55 \\
VAS r & VAS 4 fresh-tired & 0.35 & $-0.82^{*}$ & 0.30 & 0.32 \\
& VAS 5 un-concentrated & 0.17 & $0.66^{*}$ & 0.41 & -0.07 \\
\hline
\end{tabular}


Subjective feelings of activation, change in urinary aMT6 excretion, and improved and shortened sleep all seem to confirm the hypothesis that vitamin $\mathrm{B} 12$ has qualitative sleep increasing capacity (Okawa et al. 1990) with a circadian distribution toward increased wakefulness. Despite the change in sleep time, the phase of sleep is not changed as indicated by stable mid-sleep times. A genuine change of circadian phase length or position could not be demonstrated by this study design.

Increased nocturnal activity may be a direct effect of vitamin B12 and its metabolites that has not been reported before. The possibility that nocturnal activity is due to masking effects remains, as Uchiyama et al. (1995) did not find it in five healthy subjects under constant routine.

The results of the $\mathrm{d} 2$ test should be interpreted cautiously. Many participants had almost optimal scores during the pretreatment period, so that improvement was partially obscured by a ceiling effect. The increase of scores from the first to the second test on 1 day indicated a learning effect in addition to a vitamin B12 effect. The $\mathrm{d} 2$ test is designed to measure short-term concentration and is not sensitive enough to discriminate changes in normal subjects. It should therefore be replaced by long-term concentration tests in future studies.

Our temperature data do not show significant circadian changes. We decided not to interpret the nonhomogeneous results, because our study was not performed under constant routine (Dahlitz et al. 1991). Twice daily measurements of cortisol in saliva were too few to show circadian effects.

\section{CONCLUSIONS}

Our preliminary study shows that vitamin B12 in its activated form seems to be superior to cyanocobalamin in improving daytime alertness and concentration and in shortening and improving sleep in healthy persons in a dose-dependent fashion. Both forms of vitamin B12 may serve as circadian modulators of melatonin and potassium. Our findings support previous reports of a circadian effect of vitamin B12 that is mediated by melatonin, implying a possible further enhancement by light. Vitamin B12 may prove to be an alternative to stimulants when applied together with light. Its effect on sleep quality, especially on nocturnal motor activity and recuperation, should be tested by polysomnographic studies.

\section{ACKNOWLEDGMENTS}

These results are part of the second author's doctoral thesis, submitted to the Technical University of Munich. We express our thanks for the valuable contributions made by Makoto
Uchiyama, from the National Institute of Mental Health, Tokyo, Japan and the linguistic revision performed by Janet Mullington, Ottawa, Canada.

\section{REFERENCES}

Al-Ansari AAK, Perry LA, Smith DS, Landon J (1982): Salivary cortisol determination: Adaptation of a commercial serum cortisol kit. Ann Clin Biochem 19:163-166

Aldhous ME, Arendt J (1988): Radioimmunoassay for 6-sulphatoxymelatonin in urine using an iodinated tracer. Ann Clin Biochem 25:298--303

Cole RJ, Kripke DF, Gruen W, Mullaney DJ, Gillin JC (1992): Automatic sleep/wake identification from wrist activity. Sleep 15(5):461-469

Czeisler CA, Allan JS, Strogatz SH, Ronda JM, Sanchez R, Rios CD, Freitag WF, Richardson GS, Kronauer RE (1986): Bright light resets the human circadian pacemaker independent of the timing of the sleep-wake cycle. Science 233:667-671

Dahlitz M, Alvarez B, Vignau J, English J, Arendt J, Parkes JD (1991): Delayed sleep phase syndrome response to melatonin. Lancet 337:1121-1124

Honma K, Kohsaka M, Fukuda N, Morita N, Honma S (1992): Effects of vitamin B12 on plasma melatonin rhythm in humans: Increased light sensitivity phase advances the circadian clock? Experientia 48:716-720

Horne JA, Brass CG, Pettit AN (1980): Circadian performance differences between "morning" and "evening" types. Ergonomics 23:129-136

Kamgar-Parsi B, Wehr T, Gillin C (1983): Successful treatment of human non-24-hour sleep-wake syndrome. Sleep 6(3): 257-264

Laudat K, Cerdas S, Fournier C, Guiban D, Guilhaume B, Luton P (1988): Salivary cortisol measurement: A practical approach to assess pituitary-adrenal function. J Clin Endocrinol Metab 66:343-348

Maeda K, Okamoto N, Nishimoto M, Hoshino R, Ohara K, Ohashi Y, Kawaguchi K (1992): A multicenter study of the effects of vitamin B12 on sleep-waking rhythm disorder: In Shiuzuoka prefecture. Jpn J Psychiatry Neurol 46(1):229-230

Monteleone P, Maj M, Fusco M, Orazzo C, Kemali D (1990): Physical exercise at night blunts the nocturnal increase of plasma melatonin levels in healthy humans. Life Sci 47:1989-1995

Ohta T, Ando K, Iwata T, Ozaki N, Kayukawa Y, Terashima M, Okada T, Kasahara Y (1991): Treatment of persistent sleep-wake schedule disorders in adolescents with methycobalamin (vitamin B12). Sleep 14(5): 414-418

Okawa M, Mishima K, Nanami T, Shimizu T, Iijima S, Hishikawa Y, Takahashi K (1990): Vitamin B12 treatment for sleep-wake rhythm disorders. Sleep 13(1):15-23

Pollmächer T, Schulz H (1987): The relation between wristactiraphic measures and sleep stages. In Chase $\mathrm{MH}$, McGinty DJ, O'Connor C (eds), Sleep Research. Los Angeles, CA, UCLA Brain Information Service/Brain Research Institute 16:55

Sadeh A, Alster J, Urbach D, Lavie P (1989): Actigraphically 
based automatic bedtime sleep-wake scoring: Validity and clinical applications. J Ambul Monitoring 2:209-216

Souêtre E, Salvati D, Belugou JL, Krebs B, Darcourt G (1989): 5-Methoxypsoralen increases evening sleepiness in humans: Possible involvement of the melatonin secretion. Eur J Clin Pharmacol 36:91-92

Takahashi K (1992): A multicenter study on sleep-wake rhythm disorders in Japan: Preliminary results. Jpn J Psychiatry Neurol 46(1):231-232

Test d2 Aufmerksamkeits-Belastungs-Test (1978): Verlag für Psychologie. Hogrefe CJ (ed), Göttingen

Uchiyama M, Mayer G, Okawa M, Meier-Ewert K (1995):
Effects of vitamin B12 on circadian body temperature rhythm. Neurosci Lett 192(1):1-4

Waldhauser F, Saletu B, Trinchard-Lugan II (1990): Sleep laboratory investigations on hypnotic properties of melatonin. Psychopharmacology 100:222-226

Wever RA (1979): The Circadian System of Man. Springer Verlag, New York

Yamazaki J, Sugishita M, Moriya Y, Isojima G, Ohshima H, Yamazaki O, Takeda Y, Yamauchi T, Takashima M, Takahashi K (1991): The effects of vitamin B12 on the suppression of melatonin secretion under illumination. Jpn J Psychiatry Neurol 45(1):169-170 